\title{
Observations on glial inclusion bodies in a case of acute disseminated sclerosis
}

\author{
E. J. FIELD, HENRY MILLER, AND DOROTHY S. RUSSELL ${ }^{1}$ \\ From the Medical Research Council Group on Demyelinating Diseases, King's College and \\ Royal Victoria Infirmary, Newcastle upon Tyne, and the Bernhard Baron \\ Institute of Pathology, London Hospital, London
}

SYNOPSIS An unusual rod-like structure enclosed within a vacuole is described as occurring in $\frac{\vec{\omega}}{\omega}$ enlarged glial cells associated with the lesions encountered in an uncommonly acute case of multiple i sclerosis apparently heralded by an attack of 'viral encephalitis'. Similar bodies were not found in $\infty$ a variety of other enlarged glial cells. An encapsulated 'grape-fruit' like structure was also seen. 옥

Various fragmentary cytoplasmic inclusions in glial cells have been described by several neuropathologists in the past (e.g., Anton and Wohlwill, 1912), but those found recently at necropsy of an unusual case of disseminated sclerosis presented a morphological constancy worthy of record. When they were observed a search was made in enlarged glial cells encountered in other neurological conditions, but nothing comparable was seen. The special glial inclusions observed in this case of acute disseminated sclerosis are described in the hope that other observers will be stimulated to undertake a careful search of suitable material.

\section{CLINICAL HISTORY}

A 39-year-old woman's medical history began in December 1955 when she developed an acute 'influenzal' illness characterized by vomiting, headache, photophobia, vague pains in the limbs, slight fever, and some drowsiness. There were no focal neurological signs, but the spinal fluid contained $40 \mathrm{mg}$. of protein and 80 cells per c. ml., of which 20 were polymorphonuclear leucocytes and the remainder lymphocytes. On these findings a viral encephalitis was suspected, though no virus studies were undertaken. The patient recovered from the acute illness within 14 days but remained depressed and slept badly. In February 1956 persisting depressive symptoms were investigated by a neurosurgeon: an electroencephalogram and skull radiograph were normal and the patient was treated with physiotherapy. There was some slight improvement, but in October 1956 a psychiatrist advised electroconvulsive therapy for her persisting depression.

Received for publication 1 December 1961.

${ }^{1}$ Present address: Holcombe End, Westcott, Dorking, Surrey.
This was declined, and the psychiatric condition con- $\vec{\varphi}$ tinued unchanged until April 1957, when the patient's o husband had a severe illness and her condition became frankly psychotic. In May 1957 she finally accepted a recommendation to have electroconvulsive treatment, which was immediately followed by striking improvement. Three months later, however, severe depression recurred and in view of the past history she was re-admitted to $\mathbb{D}$ hospital, on this occasion under the care of a neurologist. Examination of the nervous system was entirely negative, $\vec{\partial}$ and the depressive symptoms rapidly responded to a second course of electrical treatment. On this occasion depression did not recur, but within a few weeks the patient noticed occasional unsteadiness on her feet. This increased, and in November 1957 she first complained of $?$ clumsiness of the right hand and some change in her handwriting. Over the course of a few weeks she developed the sensation of a tight band round both legs below the $ᄋ$ knees, and round the right forearm below the elbow, with $₹$ cold and tingling feelings in these three limbs. Walking 0 became difficult and demanded concentration. Although these physical symptoms were unaccompanied by any $\frac{7}{0}$ recurrence of depression they were attributed by her family to emotional disturbance, and she was not referred $N_{\sigma}$ for neurological re-assessment until January 1958. On this occasion examination revealed fine phasic nystagmus $O$ on looking to the left and marked clumsiness of the right ${ }_{\omega}^{N}$ upper limb with astereognosis and impaired two-point $O$ discrimination in the right hand. Similar but less conspicuous findings were evident in the left hand. The abdominal reflexes were absent and the deep reflexes $\stackrel{\Phi}{\rightarrow}$ grossly increased in both legs with ankle clonus, extensor :plantar responses, and dense loss of vibration sense up to the iliac crests. In retrospect it seemed almost certain $\vec{D}$ that the unequivocal disseminated sclerosis had been $ᄋ$ heralded by the evanescent encephalitic illness of two $\mathbb{D}$ years ago. Within about a month the patient considerably $\sigma$ improved but recovery was incomplete and she remained 
a semi-invalid, walking with difficulty. Exactly 12 months later the gradual onset of blurred vision first in the left and then in the right eye, with acute deterioration of gait and increasing clumsiness of the hands, led to her readmission to hospital. At this stage examination showed optic atrophy on the left and acute retrobulbar neuritis with slight swelling of the optic disc on the right. Reflexes in the upper limbs were now strikingly increased, and a spastic quadriparesis was accompanied by profound ataxia with great difficulty in walking or standing. There were no emotional disturbances. The spinal fluid was normal except for a marked increase in gamma globulin which constituted $30 \%$ of a total protein of $46 \mathrm{mg}$. per $100 \mathrm{ml}$. Undramatic physical improvement was observed during three weeks' treatment with corticotrophin, but this was followed by the development of an acute agitated depression. This was of frankly psychotic pattern and responded rapidly to imipramine (Tofranil) which she took for the remainder of her life with continuing relief of the psychiatric symptoms. However, during the first nine months of 1959 she remained very severely disabled, confined to bed and complaining bitterly of various discomforts in her limbs and of continuous burning pain in the lumbar region. There was no remission and, in the middle of September 1959, she developed a severe exacerbation beginning with intense pain and rigidity in the occipito-cervical region, which was followed by total paralysis of the legs, double incontinence, progressive flaccid weakness of both arms, and profound loss of sensation up to the mid-thorax. Dysphagia became complete, dysarthria progressed to anarthria, stupor deepened to coma and was accompanied by progressive respiratory and peripheral circulatory failure. Death occurred five days after the onset of this terminal episode.

\section{POST-MORTEM EXAMINATION}

Necropsy was limited to the brain and upper spinal cord, and was performed four hours after death. No unusual features were observed on naked-eye examination.

In order to obtain material for virus studies the brain was sectioned in the fresh state. A large yellow, plaque-like area was seen in the floor of the fourth ventricle and appeared to extend about half way through the thickness of the pons. Similar discrete yellowish areas were scattered throughout the cerebral hemispheres, one particularly striking and well-defined lesion, about $1 \mathrm{~cm} . \times 1 \mathrm{~cm}$., being located in the white matter of the right frontal lobe.

Material from the margins of these naked-eye lesions together with adjacent white matter was removed in as sterile a manner as possible and prepared at once as a $20 \%$ homogenate in saline. This was used for the inoculation of animals and tissue cultures. The greater part of the brain was preserved at $-20^{\circ} \mathrm{C}$., but representative areas were fixed in $10 \%$ formol saline and embedded in paraffin.
The optic chiasma and nerves were cut longitudinally.

Sections were stained by haematoxylin and eosin, phosphotungstic acid haematoxylin, van Gieson, the periodic-acid-Schiff method, Giemsa, Gram, and Macchiavello's stains. Myelin was stained by Loyez' method. Frozen sections were also made from representative plaques and stained for fat.

All plaques showed signs of recent activity. They contained large numbers of compound granular corpuscles and were associated with a minor degree of perivascular cuffing by lymphocytes. The lesion observed with the naked eye in the right frontal white matter showed the Balo concentric phenomenon. Only in the optic nerves were lesions mainly sclerotic. There they seemed older and were characterized by greatly enlarged glial cells, many of which presented convoluted nuclei almost suggestive of neoplasm.

Within the cytoplasm of these markedly enlarged astroglial cells oil-immersion examination showed the frequent occurrence of a rod-like structure within a vacuole. Figs. 1 to 3 show its morphological features. In Fig. 1 it is orientated longitudinally; in Fig. 2 it has been cut transversely; in the upper right cell of Fig. 3 it is caught obliquely, and below two rod-like structures is contained within a 'binucleated' cell, the left being cut transversely and the right longitudinally. Numerous examples of the same structure were present in these enlarged glial cells of the optic nerves. As a rule only one was present in each cell, but occasionally two (though never more) were seen in a particularly large cell with a complicated nucleus. These bodies were not haematoxylinophilic and did not stain with Schiff's reagent, Giemsa, or Macchiavello's method. They were best examined in phosphotungstic-acid-haematoxylin-stained preparations with the use of a green filter. Without the filter they stained a pale pinkish colour almost identical with that of the astrocytic cytoplasm. In this they were quite distinct from blepharoplasts. Once recognized under oilimmersion they could readily be identified with the $4 \mathrm{~mm}$. lens. Much care was expended in determining the exact relation of these structures to the cytoplasm: it was concluded that they were actually within it, and continuity with fibrils outside the cells was never observed.

In other regions of the nervous system these rodlike bodies were much fewer and also smaller. In general they were larger and more conspicuous in greatly enlarged glial cells, suggesting that they might originate in hypertrophied normal intracytoplasmic apparatus. This possibility will be discussed below.

There was no evidence of a burned-out antecedent encephalitis. 

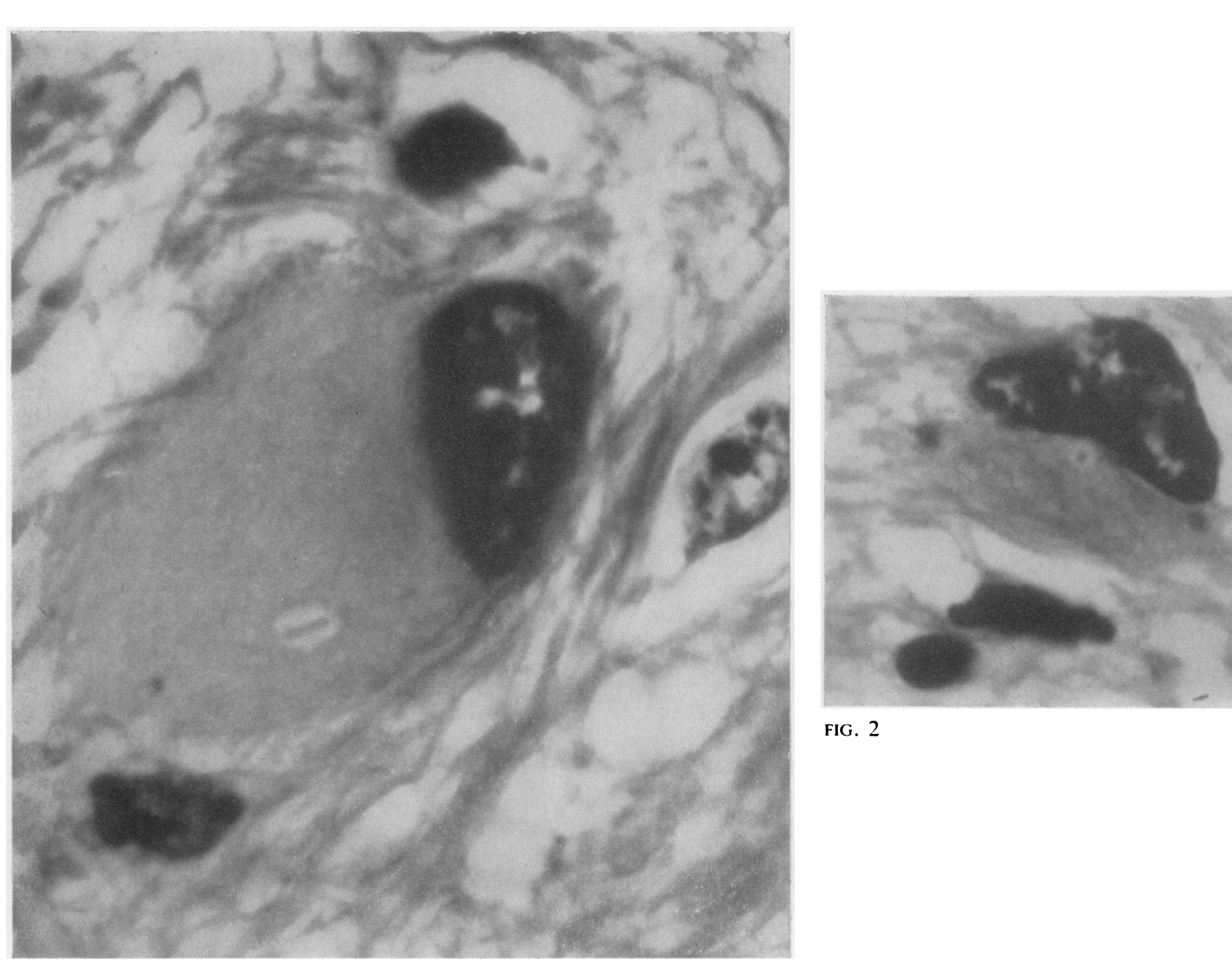

政

FIG. 1

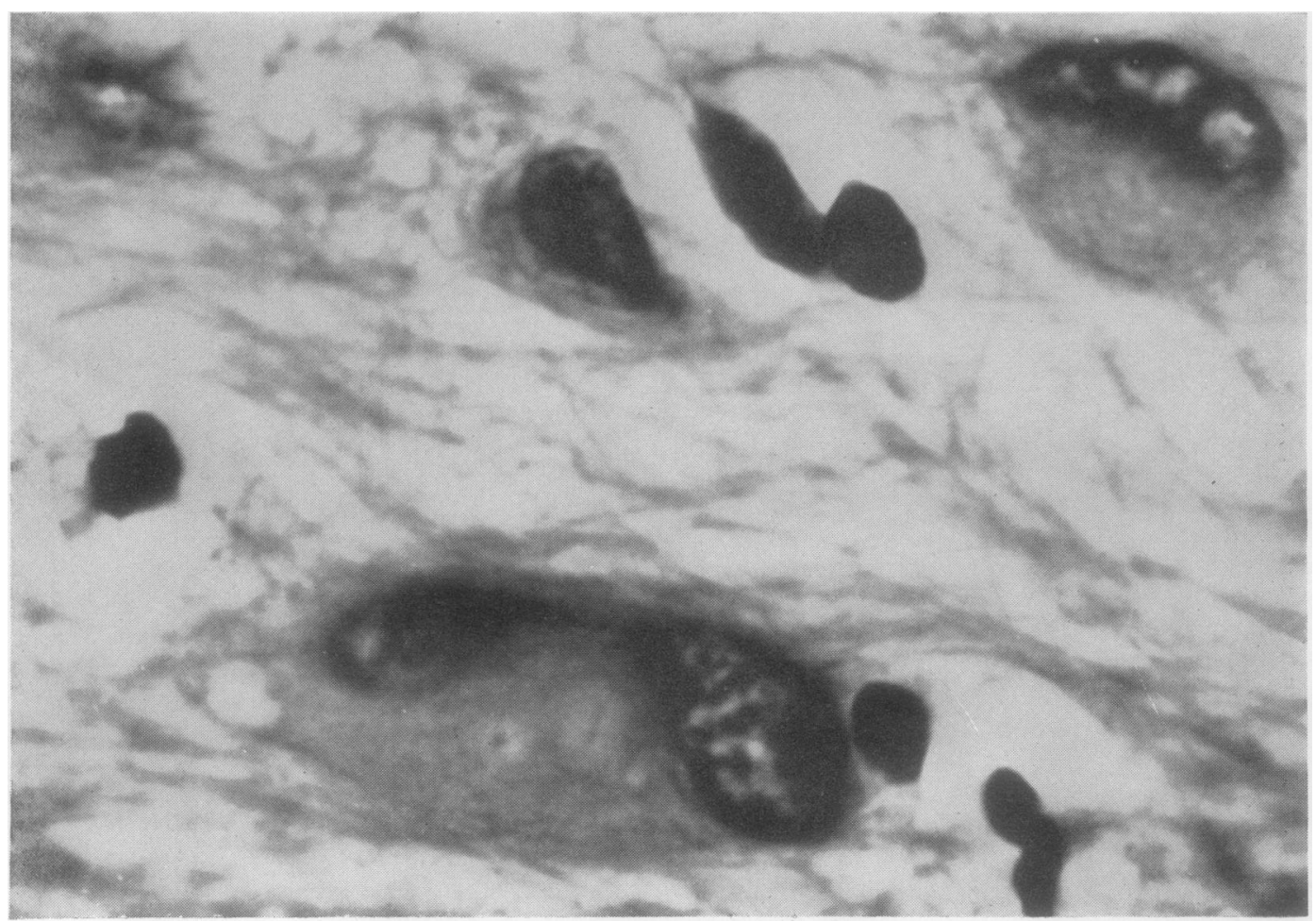

FIG. 3 


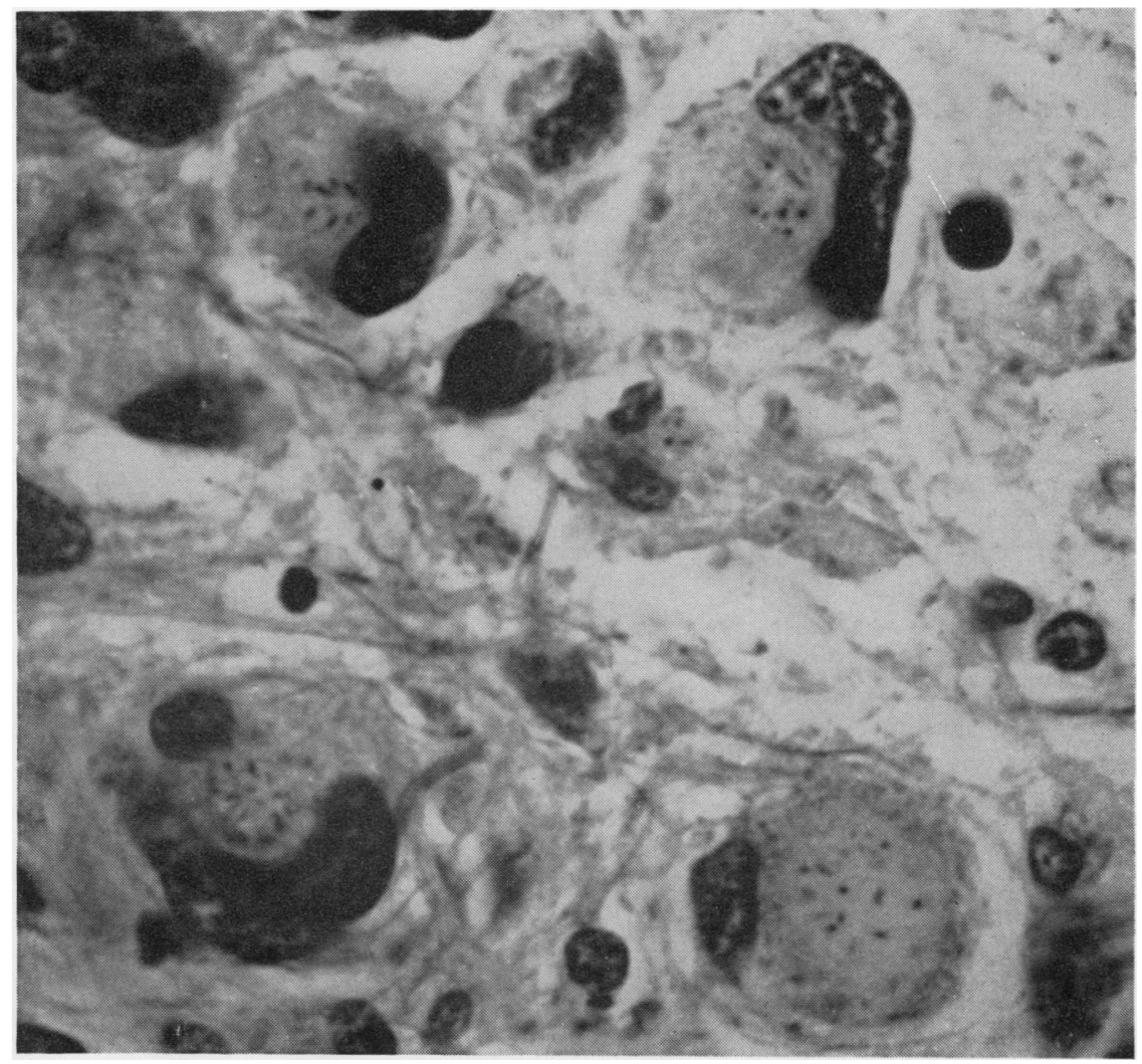

FIG. 4

FIG. 1. Acute multiple sclerosis. Greatly enlarged glial cell from optic nerve. Rod-like structure, apparently cut longitudinally, contained within a vacuole. P.T.A.H. $\times$ 1,330 .

FIG. 2. Glial cell from same region as in Fig. 1. Rodlike structure cut transversely contained within vacuole. P.T.A.H. $\times 2,250$.

FIG. 3. Same region as in Figs. 1 and 2. In the upper right corner a glial cell contains a rod-like body orientated obliquely within a vacuole. Below a binucleated cell contains two vacuoles. In the left of these a rod-like body is cut transversely and in the right longitudinally. P.T.A.H. $\times$ 2,250 .

FIG. 4. Glial heterotopia associated with von Recklinghausen's disease. Note multiple haematoxylinophilic rodlike bodies varyingly orientated within the glial cells; they are not contained within individual vacuoles. P.T.A.H. $\times 1,160$.

FIG. 5. Glial heterotopia (as in Fig. 4). Multinucleated glial cell showing several rod-like haematoxylinophilic bodies apparently contained within a common vacuole. P.T.A.H. $\times 2,250$.

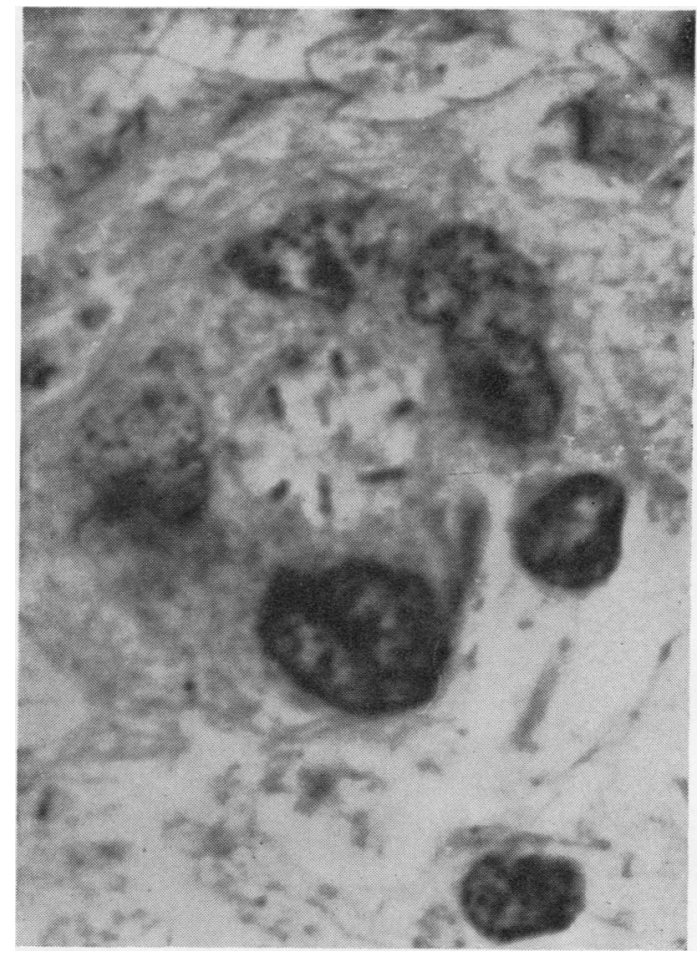

FIG. 5 


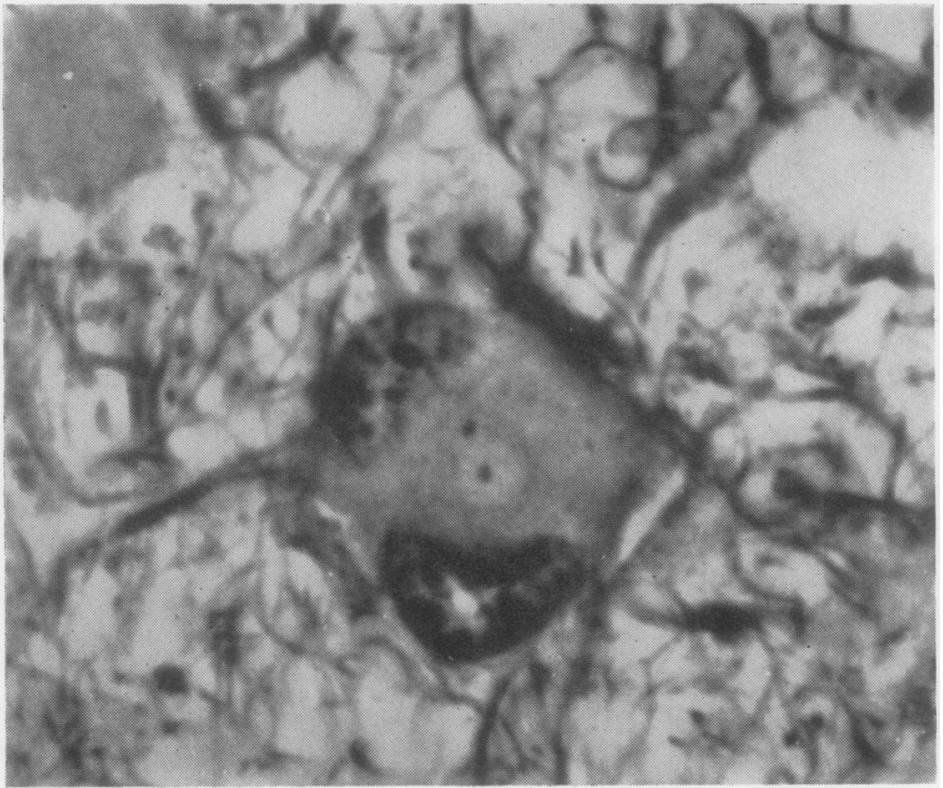

FIG. 6
FIG. 6. Schilder's disease. Glial cell. showing two punctate structures, one within a vacuole and the other without. These structures were haematoxylinophilic and did not occur in rod forms. P.T.A.H. $\times 2,250$.

FIG. 7. Same case as in Fig. 6. Vacuo- ָొ lation in glial cells. P.T.A.H. $\times 2,250 .+$

FIG. 8. Acute multiple sclerosis. Coccoid bodies, some resembling diplo-cocci and contained within vacuoles, in̄응 enlarged glial cell cytoplasm. P.T.A.H. $\times 2,250$.

FIG. 9. Acute multiple sclerosis. Un- $-\infty$ identifiable structure having a 'halved-o grapefruit' appearance found in the edge of an acute lesion of Balo type. The $\vec{z}$ punctate masses of haematoxylinophilic material are different from the rod-like bodies of Figs. 1 to 3.P.T.A.H. $\times 2,250 . \overrightarrow{0}$

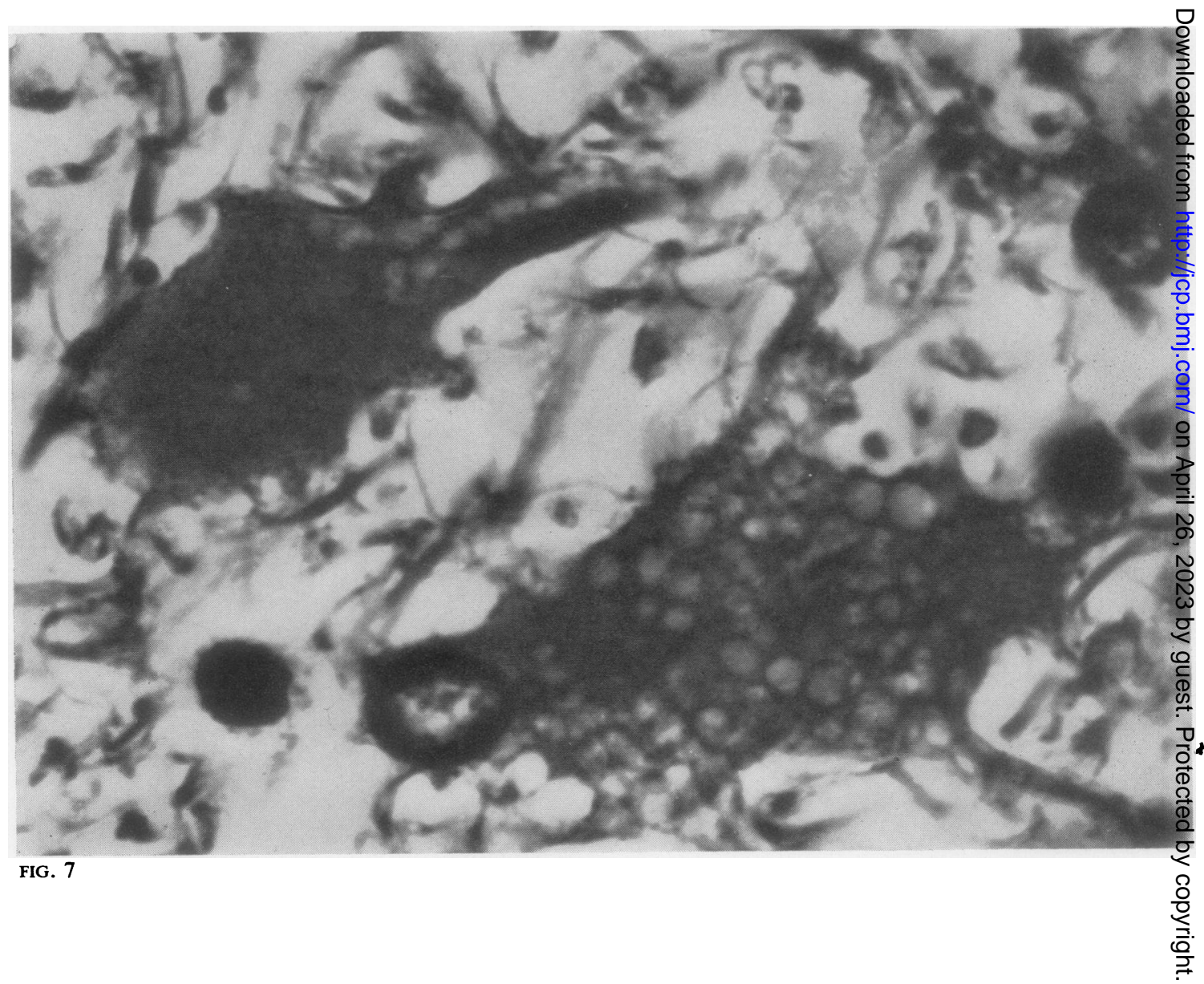




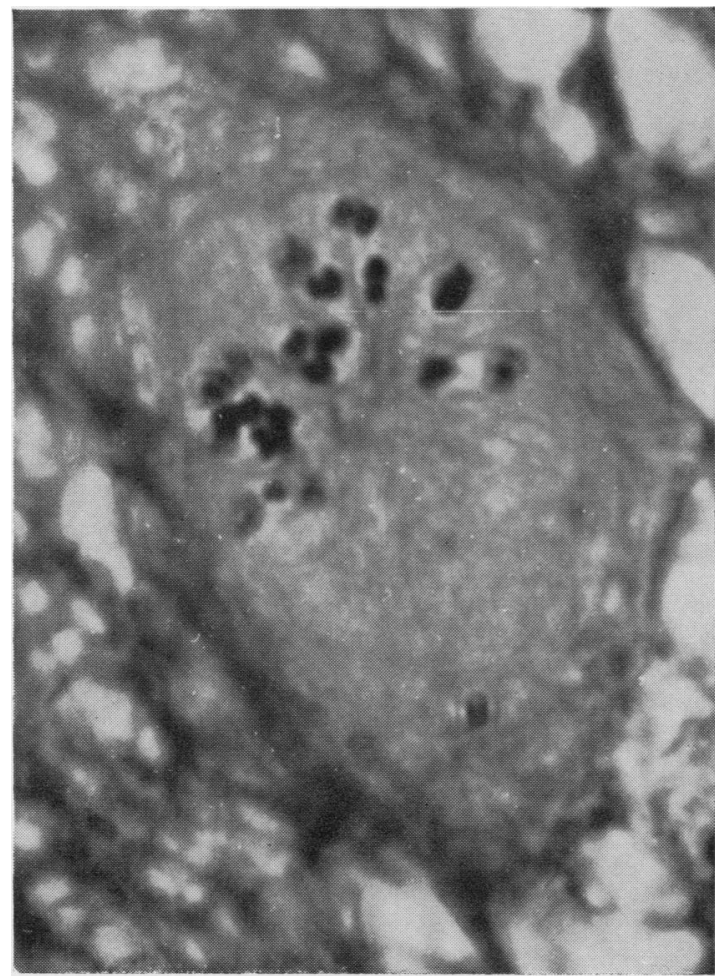

FIG. 8

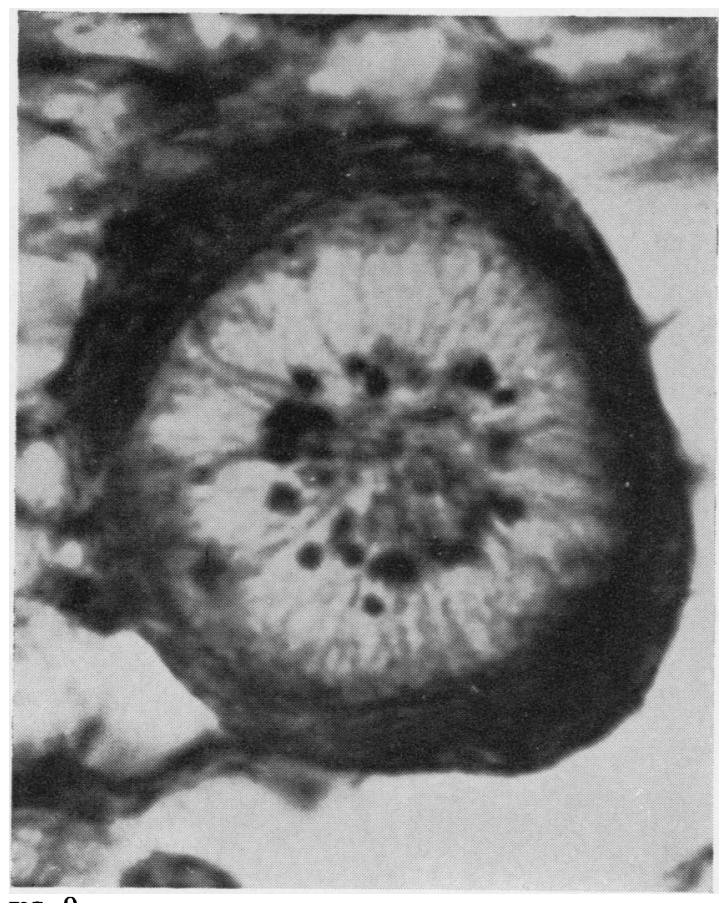

OBSERVATIONS IN OTHER MATERIAL

The enlarged glial cells which occur in a variety of other conditions were searched for similar inclusions. These conditions included 'glial heterotopias' in a case of von Recklinghausen's disease (Russell and Rubinstein, 1959), cortical atrophy with gliosis following presumed soluble barbitone (Medinal) poisoning, post-irradiation sclerosis, giant-cell glioblastoma, tuberose sclerosis, and Schilder's disease. In this material many of the irregular inclusion particles, sometimes associated with multiple vacuolation, described by the older German neuropathologists (Alzheimer, 1910; Anton and Wohlwill, 1912) were readily identified but nothing was seen resembling the rod-like structures of the disseminated sclerosis sections.

The 'glial heterotopias' described by Russell and Rubinstein (1959) provided some striking inclusions in addition to the blepharoplasts they illustrated (Figs. 19 and 20, p. 35 loc. cit.). Unlike the multiple sclerosis inclusions, those in the glial heterotopias were haematoxylinophilic and were commonly arranged in clusters (Fig. 4). Occasionally these clusters of deeply stained rod-like inclusions appeared to be enclosed within a limiting membrane (Fig. 5).

Schilder's disease presented nothing comparable with the rod-like structures seen in the disseminated sclerosis preparations. Occasional dot-like structures sometimes contained within a halo were found but no rods (Fig. 6). This material also showed some glial cells with a vacuolar degeneration of cytoplasm (Fig. 7).

\section{DISCUSSION}

The significance of the non-haematoxylinophilic elongated structures seen in this case of acute disseminated sclerosis is obscure. They do not stain by the usual methods for viral inclusion bodies. The punctate appearance seen in Fig. 2 suggests a centrosome, but no elongated forms of the latter have been described and this interpretation seems unlikely. Nor do they resemble the 'kokkenähnlich angeordnete Chromatinteilchen' referred to by Peters $(1935,1958)$. Indeed one or two instances of the latter were seen in our case (Fig. 8). A further unidentifiable structure was seen (Fig. 9) which seemed to be an encapsulated formation within which thread-like, radially arranged filaments were connected to coccoid-like particles at their inner ends. They were very rare. In addition to that shown in Fig. 9 only one other doubtful example was found. They occurred in the active margin of an acute Balo type of lesion in the frontal white matter. The whole structure appeared to be an enlarged glial 
cell largely occupied by the ray and coccoid-like formation. The whole appeared enclosed in a concentrically laminated 'capsule'. No trace of nucleus could be found on fine focusing and the strongly haematoxylinophilic particles may be presumed of nuclear origin. Whether the 'capsule' was a true one or a peripheral cytoplasmic remnant was uncertain. Peters (1935) seems to have noted similar structures in an acute case of 'disseminated non-purulent encephalomyelitis' of six weeks' duration. He found, in a few of the cells which contained coccoid-like chromatin particles, a ray-like figure from the centre to the periphery of the cell (eine strahlige Figur vom Zentrum zur Peripherie der Zelle), so that the whole cell on cross section looked like a cut lemon, a description which accords well with the appearances in Fig. 9, though he does not mention the 'capsule' seen there. He was struck by the resemblance of these structures to parasites but found they did not stain by Ziehl-Neelsen, Gram, or methylene blue. Like ourselves he was not able to draw any conclusion as to their nature but remarked on their occurrence in the marginal proliferating zone of lesions. His case was very acute, and lesions were florid as in our own material.

Organisms of one sort or another have not infrequently been described in the lesions of disseminated sclerosis. On the evidence available the authors would not wish to add to them, especially since all attempts to establish disease by the inoculation of animals such as guinea-pig, rat, mouse (both adult and suckling), chicken (adult and newly hatched), monkey, and sheep were unsuccessful, as were growths attempted in Hela cells and monkey kidney.

The rod-like structures do not correspond to any normal cell component known to the authors, which might have hypertrophied in the large glial cells $\frac{\text { : }}{\overrightarrow{7}}$ Moreover they have not been reported in electron? microscopic studies of reacting astroglia in the vicinity of experimental brain wounds.

The only suggestion offered is that careful high? power examination should be made in every lesion of acute multiple sclerosis so that these structures $\frac{\text { क }}{\sqrt{6}}$ if present, may be identified and their relations to̊ other pathological findings clarified.

We would like to thank Miss G. Joyce for histologica preparations of the multiple sclerosis material.

\section{REFERENCES}

Alzheimer, A. (1910). In Histologische und histopathologische Arbeiten $\vec{r}$ über die Grosshirnrinde, ed. F. Nissl and A. Alzheimer, Vol. 3 p. 410. Fischer, Jena.

Anton, G., and Wohlwill, F. (1912). Z. ges. Neurol. Psychiat. (Orig.) $12,31$.

Peters, G. (1935). Ibid., 153, 356.

(1958). 'Multiple Sklerose' and 'Encephalomyelitis disseminata' non purulenta acuta (Akute multiple Sklerose)'. Handbuch der speziellen pathologischen Anatomie und Histologie, ed. $\mathrm{O} \backslash$ Lubarsch, F. Henke and R. Rössle, band XIII, pp. 525-62990
Zweiter Teil. Bandteil A. Springer, Berlin.

Russell, D. S., and Rubinstein, L. J. (1959). Pathology of Tumours oto the Nervous System. Arnold, London.

\section{ADDENDUM}

Since this manuscript was submitted sheep injected़ intracerebrally by Dr. P. A. Pálsson of the Institute of Experimental Pathology, Keldur, Reykjavik, witl? a suspension of the brain described in this paperō have developed after a long incubation period $\overrightarrow{\mathrm{z}}$ disease which is histologically indistinguishable fron scrapie. Further experiments have been set up to. confirm this altogether unexpected result, reporte here in the hope that independent investigations will be made. 